\title{
Biliary Colic Preceding Acute Gallstone Pancreatitis
}

\author{
Alejandro Oría • Gustavo Kohan
}

Received: 12 March 2009/Accepted: 3 June 2009 / Published online: 23 June 2009

(C) 2009 The Society for Surgery of the Alimentary Tract

\section{Dear Sir,}

We read with much interest the article by Besselink et al. ${ }^{1}$ on the "warning" episodes of biliary colic preceding "complicated" gallstone disease. Up to $58 \%$ of patients with acute gallstone pancreatitis had experienced episodes of biliary colic prior to onset of the attack. Patients' delay or reluctance of the general practitioner to refer the patient for imaging studies or specialist consultation precluded timely treatment of gallstone disease.

Biliary colic is a poor descriptor for biliary pain due to "uncomplicated" gallstone disease. Although biliary colic is most commonly due to cystic duct obstruction, identical pain may be secondary to main bile duct obstruction. In the absence of jaundice, differentiation between cystic duct obstruction due to gallbladder gallstones and main bile duct obstruction due to choledocolithiasis cannot be made without radiologic and laboratory studies. A clear distinction between these two conditions is important for the prevention of acute gallstone pancreatitis.

Gallstone migration through the papilla of Vater is the triggering event for acute gallstone pancreatitis. We investigated gallstone migration in 39 patients admitted to the hospital with upper abdominal pain due to gallstone disease and a distal bile duct measuring $7 \mathrm{~mm}$ or more on ultrasound examination. $^{2}$ None of the patients had acute pancreatitis or acute cholangitis. Using stool screening and ultrasound monitoring for diameter changes of the biliary and pancreatic

A. Oría

Av Alvear 1583, 1014 Capital,

Buenos Aires, Argentina

e-mail: aleoria@fibertel.com.ar

G. Kohan $(\bowtie)$

Department of Surgery, Cosme Argerich Hospital,

University of Buenos Aires,

Buenos Aires, Argentina

e-mail: gustavokohan@yahoo.com.ar duct, migration of small gallstones was detected in ten patients; of these, six exhibited total serum bilirubin values below $2 \mathrm{mg} / \mathrm{dL}$ throughout migration. All of the six patients had experienced recent episodes of "biliary colic" and had been treated symptomatically elsewhere. This high prevalence of anicteric episodes of gallstone migration was confirmed in another study using a greater number of patients. ${ }^{3}$ In contrast to a widely held view, gallstone migration does not occur after a brief impaction of the stone at the distal bile duct. Even the smallest stone may obstruct the upper segment of the sphincter for several days or even weeks before reaching the papilla. ${ }^{2}$ In order to prevent acute gallstone pancreatitis, early identification of patients undergoing anicteric episodes of gallstone migration is essential. At present, however, many of these patients are labeled "biliary colic" and managed on an outpatient basis.

We agree with Besselink et al. ${ }^{1}$ on the importance of increasing awareness of the general public and the general practitioner for the warning aspect of upper abdominal pain. It is our opinion, however, that another important issue is the use of the term biliary colic to describe any episode of upper abdominal pain due to presumed uncomplicated gallstone disease. Biliary colic is a confounding term that should be substituted for a precise descriptor of the site of biliary obstruction. In the present evidence-based medicine era, inaccurate terms should be removed from medical language. ${ }^{4}$

\section{References}

1. Besselink MG, Venneman NG, Go PM, et al. Is complicated gallstone disease preceded by biliary colic? J Gastrointest Surg 2009;13:312-317. doi:10.1007/s11605-008-0729-y.

2. Oría A, Frider B, Alvarez J, et al. Biliary and pancreatic obstruction during gallstone migration. Int J Pancreatol 1988;3:157-164.

3. Oría A, Alvarez J, Chiappetta L, et al. Risk factors for acute pancreatitis in patients with migrating gallstones. Arch Surg 1989;124:1296-1296.

4. Shanahan F, Quigley EMM. Bad language in gastroenterology. Curr Gastroenterol Rep 2008;16:91-93. doi:10.1007/s11894-008-0026-7. 